\title{
Muscular Function: An alternative way to identify cognitive impairment, Secondary Analysis From SABE-Colombia
}

Elkin Garcia-Cifuentes ( $\nabla$ elkingarciaci@gmail.com )

Pontificia Universidad Javeriana

Felipe Botero-Rodríguez

Pontificia Universidad Javeriana

Felipe Ramirez Velandia

Pontificia Universidad Javeriana

Angela Iragorri

Pontificia Universidad Javeriana

Isabel Marquez

Pontificia Universidad Javeriana

Geronimo Gelvis

Pontificia Universidad Javeriana

Maria-Fernanda Acosta

Pontificia Universidad Javeriana

Alberto Jaramillo-Jimenez

Stavanger University Hospital

Francisco Lopera

University of Antioquia

Carlos Cano-Gutierrez

Pontificia Universidad Javeriana

\section{Research Article}

Keywords: gait speed, handgrip strength, cognitive impairment, biomarker, preclinical dementia, motor dysfunction

Posted Date: December 23rd, 2020

DOI: https://doi.org/10.21203/rs.3.rs-131443/v1

License: @ (i) This work is licensed under a Creative Commons Attribution 4.0 International License. Read Full License

Version of Record: A version of this preprint was published at Alzheimer's \& Dementia on December 1st, 2021. See the published version at https://doi.org/10.1002/alz.050392. 


\section{Abstract \\ Background}

Traditionally, the identification of cognitive impairment is based on neuropsychological tests and supported with not widely available biomarkers. This study aimed to establish the association between motor function (Gait Speed and Handgrip Strength) and the performance in a global cognitive performance and various cognitive domains. Our secondary objective was to determine a cut-off point for Gait Speed and Handgrip Strength to classify older adults as cognitively impaired.

\section{Methods}

This is a secondary analysis from the SABE Colombia study (Health, Well-Being, and Aging) conducted in 2015. We performed linear regression models, to establish association with motor function, clinical, and sociodemographic variables, and predict the scores of the Mini-mental State Examination and its domains (i.e. orientation, recall, counting, and language). The evaluation of the motor function variables as an instrument to separate cognitively impaired older adults was evaluated by developing a receiving operating characteristic curve (ROC).

\section{Results}

Gait speed was associated with orientation $\left(r^{2}=0.16\right)$, language $\left(r^{2}=0.15\right)$, recall memory $\left(r^{2}=0.14\right)$ and counting $\left(r^{2}=0.08\right)$. Similarly, handgrip strength was associated with orientation $\left(r^{2}=0.175\right)$, language $\left(r^{2}=0.164\right)$, recall memory $\left(r^{2}=0.137\right)$, and counting $\left(r^{2}=0.08\right)$. Slow gait had a cut-off point of $0,59 \mathrm{~m} / \mathrm{s}$, with an area under the curve (AUC) of 0.629 (0.613-0.646), whereas a weak handgrip strength had an AUC of 0.653 (0.645-0.661), with a cut-off point of $17.50 \mathrm{Kg}$ for separating those older adults with cognitive impairment.

\section{Conclusions}

Gait Speed or Handgrip Strength are similarly associated with cognitive performance, exhibiting the larger associations with orientation and language domains. Gait Speed and Handgrip Strength can be easily performed by any clinician and seems to be useful screening tools to detect cognitive impairment.

\section{Background}

Dementia has become a worldwide health priority that affects the quality of life in older adults and caregivers (1). Research in this area is particularly important in Colombia where the largest cohort worldwide with familiar autosomal dominant Alzheimer's disease due to PSEN1-E280A and PSEN1-I416T genetic variants have been reported $(2,3)$. In addition, as a middle-income country, smoking, alcohol consumption, diabetes, hypertension, lack of education, and limited access to the healthcare system increase the risk of dementia by multifactorial causes(4). The latter can be challenging for a timely diagnosis of dementia in the Colombian context, as the identification of cognitive impairment has been traditionally based on neuropsychological tests and supported by not widely available biomarkers. Thus, early identification of cognitive impairment through non-cognitive biomarkers is an emerging approach that can be useful to set up preventive strategies in dementia in low and middle-income countries $(5,6)$.

There is growing evidence suggesting that dementia, particularly Alzheimer's Disease (AD), is a continuum with a long preclinical stage that can be reflected in early motor symptoms (7-9). In recent years, the association between cognitive functions and motor function has been elucidated, suggesting shared neural networks substrate including frontal-hippocampal pathways (10). Impairment in any of these circuits can be reflected in motor or cognitive disturbances. Thus, motor function assessment can be a useful correlate of cognition and a promising predictor of Mild Cognitive Impairment (MCl) and dementia (5).

In the Colombian context, there are previous publications evidencing that motor disfunction are associated with dementia(11, 12). The 5th Canadian Consensus Conference: This expert panel suggested that individuals with subjective memory complaints, motor dysfunction (i.e. gait speed disturbances, and dual-task gait impairment), are prone to developing cognitive decline and may be closely followed and screened (13). Currently, no standardized screening protocols are using those non-cognitive markers that can be applied to Colombia.

In line with the above, the assessment of motor function with Handgrip strength (HS) evaluation has shown to be a good indicator of the whole muscular strength (14), and preliminary reports have suggested that HS might be a good predictor for the risk of developing cognitive decline(11), frailty, and dependency in instrumental activities of daily living $(15,16)$. Another measure of muscular function that has been associated with cognitive impairment is the gait speed (GS) $(17,18)$. Abnormalities in GS precede cognitive decline by several years $(19-21)$ and have been considered a potential novel biomarker of $\mathrm{MCl}$, $A D$, and other dementias. (22). Both HS and GS are easy to perform, objective, non-invasive, low-cost, widely available, and safe measures, increasing their acceptability and generalisability. However, little is known about which of the cognitive domains are predominantly related to motor performance, even if that can be valuable when performing a clinical evaluation in the elderly.

Therefore, the purpose of the following study was to establish the association of motor function (GS and HS) with cognitive function, assessed with the Minimental State Examination (MMSE) and its domains (Orientation, Calculation, Language, and Memory). Our secondary objective was to determine a cut-off point for GS and HS to classify older adults as cognitively impaired. Based on our preliminary reports(11, 12), we hypothesise that reduced motor function might be associated with the global cognition and some domains such as orientation and memory. 


\section{Methods \\ Study design}

This is a secondary analysis from the SABE Colombia study (Health, Well-Being, and Aging) conducted in 2015. SABE Colombia is a population-based crosssectional study on health, aging, and well-being. SABE Colombia study included a country representative sample of 23,694 older adults living in the community aged 60 years or more. The sample was probabilistic, clustered, stratified/multi-staged by urban and rural areas. The methods and procedures conducted in the study were based on those used in the SABE international multicenter study in order to reach comparability, generalizability, and harmonized protocols; however, it was adapted to the Colombian context. The information was integrated within the general framework of the Colombia National Surveys System. Other technical details of the SABE Colombia study can be found elsewhere (23).

For the current analyses, we included a subsample of 5381 participants. A detailed flowchart with eligibility criteria and selection of our subsample is included, please see Fig. 1.

\section{Variables}

\section{Gait Speed}

GS was computed from one sub-test of the Short Physical Performance Battery (SPPB), previously validated for the Colombian population applied in the SABE Colombia study(24). The participants were asked to walk 3 meters at their regular pace two times from a standing position. Following SPPB protocols, the best timing of both trials was used to determine GS and previous 3 meters GS was used in Colombian SABE(12).

\section{Hand Grip Strength}

HS was measured using an adjustable digital handgrip dynamometer (Takei Scientific Instruments Co., Tokyo, Japan). Participants were instructed by the interviewer. The best score for each hand was recorded in $\mathrm{Kg}$. The handgrip strength was calculated as the average of the left and right hand(25).

\section{Functional status}

For basic activities of daily living, we used the Barthel index (ranging from 0 to 100), low scores imply a functional dependency (26). For instrumental activities of daily living, we used the Lawton and Brody scale (ranging from 0 to 54), high scores can be interpreted as functional impairment (27, 28).

\section{Comorbidities:}

Self-reported comorbidities or medical conditions were taken into account if had been diagnosed by a physician. The frequency of hypertension, diabetes, respiratory diseases, cardiovascular diseases, cancer, and osteoporosis was reported.

\section{Cognitive performance:}

To assess cognitive function, we used the Mini-Mental State Examination (MMSE) total score (ranging from 0 to 30), where low values indicate worse cognitive performance $(29,30)$. The MMSE was also analyzed in its cognitive domains (i.e. orientation, recall, counting, and language). We used a cutoff of MMSE score $<25$ to classify individuals as cognitively impaired following previously defined methods (31).

\section{Statistical analysis}

Given the quantitative nature of the variables, we carried out a descriptive analysis of the subsample, through the program $R$ studio. The variables assessed were: age and sex (sociodemographic) as possible cofounders; Barthel and Lawton score (functionality); MMSE and their domains (orientation, recall, counting, and language); frequency of arterial hypertension, diabetes, cancer, chronic obstructive pulmonary disease (COPD), myocardial infarction, stroke, arthropathies and mental diseases (comorbidities); finally, HS and GS (muscular function). We tested the distribution of each of these variables with the Kolmogorov-Smirnov (Lilliefors' correction) test.

\section{Association between muscular function and cognitive variables assessment}

We performed various linear regression models, establishing the cognitive variables (MMSE and its domains) as dependent variables, and the remaining variables of interest as independent variables. In accordance with our objective, we carried out the following models for each dependent variable: a) gait speed, sex, and age; b) handgrip strength, sex, and age; c) all sociodemographic, functionality variables, comorbidities, and motor function variables. In all these models we ruled out the presence of collinearity between the independent variables and evaluated possible interactions or confounding effects among the variables. For the selection of the independent variables in a final model, we used backward elimination.

\section{Muscle function for assessment of cognitive impairment}

The evaluation of the variables GS and HS as an instrument to categorize cognitive impairment was evaluated by using a sensitivity analysis. A Receiving operating characteristics (ROC) curve was made, and the Youden index was used to identify the optimal cut-off for each motor function variable. The respective areas under the curve (AUC), sensibility, specificity, and accuracy for each motor function variable was also calculated.

\section{Results}


Descriptive analysis by cognitive status is summarized in Table 1. The median age was 68 years for cognitively normal individuals and 75 years for the cognitive impaired group, and their median of schooling years was 4 years and 1 year, respectively. Women comprised $57.62 \%$ of the cognitively normal group and $62.78 \%$ of the cognitively impaired. In the muscular function, both HS and GS, were significantly lower in the cognitively impaired group when compared to those cognitively normal subjects

Table 1

Descriptive analysis by cognitive status

\begin{tabular}{|c|c|c|c|c|}
\hline & & Cognitive normal & Cognitive impaired & $P$ value \\
\hline & & \multicolumn{3}{|l|}{$\mathrm{n}(\%)$} \\
\hline Gender & Female & $2325(57.62)$ & $855(62.78)$ & 0.001 \\
\hline \multirow[t]{9}{*}{ Comorbidities } & Hypertension & $2184(54.21)$ & $843(62.17)$ & 0.001 \\
\hline & Myocardial infraction & $550(13.64)$ & $228(16.79)$ & 0.004 \\
\hline & Stroke & 149(3.7) & $89(6.55)$ & 0.001 \\
\hline & Diabetes & 657(16.34) & $240(17.69)$ & 0.251 \\
\hline & Artropaties* & $1170(29.05)$ & $343(25.33)$ & 0.008 \\
\hline & Mental Diseases** & $326(8.10)$ & 159(11.76) & 0.001 \\
\hline & Mean (SD) & & & \\
\hline & Age (years) & $68(10)$ & $75(14)$ & 0.001 \\
\hline & Schooling (Years) & $4(4)$ & $1(3)$ & 0.001 \\
\hline \multirow[t]{2}{*}{ Muscular function } & Hand Grip Strenght (kg) \$ & $23.18(8.84)$ & $18.74(8.7)$ & 0.001 \\
\hline & Gait speed (m/s) \$ & $0.723(0.23)$ & $0.586(0.23)$ & 0.001 \\
\hline \multirow[t]{2}{*}{ Functionality } & Lawton total & $0(2)$ & $4(10)$ & 0.001 \\
\hline & Barthel Index & $100(0)$ & $100(5)$ & 0.001 \\
\hline
\end{tabular}

Table 2. summarizes the demographic-adjusted linear regression models to predict cognitive variables. GS was associated with orientation $\left(r^{2}=0.16\right)$, language $\left(r^{2}=0.15\right)$, recall memory $\left(r^{2}=0.14\right)$ and counting $\left(r^{2}=0.08\right)$. Similarly, in HS with orientation $\left(r^{2}=0.175\right)$, language $\left(r^{2}=0.164\right)$, recall memory $\left(r^{2}=\right.$ $0.137)$, and counting $\left(r^{2}=0.08\right)$. Table 3 shows our final fully-adjusted model to explore the associations between motor function variables and cognitive variables. This model included age, presence of mental disorder (the only comorbidity selected after backward elimination), functionality and its interactions. The presence of these interactions implies that the magnitude of change in the cognitive variables, is even higher if both interaction variables are present. The analysis revealed that $28.9 \%$ of the variability in orientation is attributed to our described model. Also, a this model showed large effect size with language $\left(r^{2}\right.$ $=0,272)$, medium in memory recall $\left(r^{2}=0,192\right)$ and small in counting $\left(r^{2}=0,108\right)$.

Table 2

Association between motor function and cognitive domains.

\begin{tabular}{|lllllllllllll|}
\hline Measure & \multicolumn{1}{l}{ Mini Mental State Examination } & & & & & & \\
& \multicolumn{1}{l}{ Orientation } & Recall & & Counting & Language & Total & \\
\hline & R2 & p value & R2 & p value & R2 & p value & R2 & p value & R2 & p value \\
\hline Gait speed & 0.17 & $<0.001$ & 0.15 & $<0.001$ & 0.08 & $<0.001$ & 0.16 & $<0.001$ & 0.19 & $<0.001$ \\
Handgrip Strength & 0.19 & $<0.001$ & 0.15 & $<0.001$ & 0.09 & $<0.001$ & 0.18 & $<0.001$ & 0.21 & $<0.001$ \\
\hline * Both measures presented significat interaction with age & & & & & & \\
\hline
\end{tabular}

Figure 2 shows the ROC curves that evaluated HS and GS as markers of cognitive impairment. Regarding HS, the point with the largest area under the curve (AUC) was defined as $17.50 \mathrm{Kg}$, with an AUC of 0.629 (95\% Cl: $0.613-0.646)$. On the other hand, for GS a cutoff point of $0.5999 \mathrm{~m} / \mathrm{s}$ was defined, which had an AUC of 0.653 (95\% Cl: 0.645-0.661).

\section{Discussion}

Our results enhance the evidence in the relationship between muscular function and cognition. We found that motor function exhibited the largest association with the orientation domain (Final model $\mathrm{r}^{2}=0.289 ; \mathrm{GS} \mathrm{r}^{2}=0.16 ; \mathrm{HS} \mathrm{r}^{2}=0.175$ ) followed by the language domain (Final model $r^{2}=0,272 \mathrm{GS} \mathrm{r}^{2}=0.15 ; \mathrm{HS} \mathrm{r}^{2}=$ 0.164). Our findings complement preliminary reports that have showed associations between early motor function and dysexecutive symptoms and semantic memory impairments (32). 
The underlying neural mechanisms of the motor-cognition relationship is set on the place and grid cells located in the entorhinal cortex, with an important role in spatial orientation (33). Thus, a correlation between small volume of the left entorhinal cortex and muscular dysfunction was reported in those without cognitive impairment during the dual-task gait analysis (34). Previously publication determine that executive functions are essential for gait control, since gait requires the integration of sensory and perceptual information, a continuous updating of the input, and quick adaptations of the gait pattern (35-37). Similarly, a longitudinal study (38), in dual-task GS $27.4 \%$ of the variance was attributed to executive attention, while orientation and attention are cognitive domains linked to each other(38).

In the same line, speech and language are proven to be among the most reliable markers to distinguish between the types of dementia in which motor dysfunction can be observed (39). Also, speech and language are cognitive domains strongly associated with the supplementary motor area (SMA), a very important structure in motor execution, thus, alterations in the SMA and its correlated circuits (subcortical circuits, basal ganglia) may be clinically seen as alterations in language and motor performance (40). Muscle strength, specifically HS can be an overall indication of the integrity of the central nervous system and the total force of the upper limb muscles. Stronger HS is related to better performance on functional tasks as it can be an indicator of the ability to walk, rise from a chair, hold small items, toothbrush or comb (16).

Our results showed that the coefficient of variability in the cognitive domains is similar when analizing GS or HS, suggesting that both variables have similar performance when evaluating motor function correlates the cognitive status of individuals. Even though GS has emerged as one of the motor domains strongly correlated with incident dementia, our results showed that GS and HS may be an alternative parameter that can be used in the clinical practice to assess individuals at risk to develop dementia. GS and HS has been reported as a useful single marker of frailty (41). Likewise, handgrip dynamometers are inexpensive, easily portable, non-invasive, reliable, do not require extensive training, and are not biased by learning effects that can be seen in neuropsychological tests (16).

In line with our findings, previous reports have shown that poor physical performance is associated with cognitive decline. Besides, GS and HS represent a core determinant of physical frailty and sarcopenia, which are both associated with cognitive impairment (42). More recently, there has been proposed that physical and cognitive decline can occur simultaneously and they can share common etiologies (43). Hormonal levels and inflammatory biomarkers are thought to be implicated in cognitive dysfunction; indeed, hormones like Irisin, are expressed not only in the muscle but also in the brain and it reduces neuroinflammation and post-ischemic oxidative stress, suggesting that this molecule may play an important role in neuroprotection and synaptic plasticity (44). Similarly, higher levels of pro-inflammatory cytokines, such as IL-6, were associated with greater cognitive decline as well as lower HS. Associations between impaired cognitive performance and poor physical performance in GS and balance suggest that abnormalities in the nervous system processing speed may be linked to changes in cognitive function (16).

In our analyses, the accuracy of GS and HS as methods to identify cognitive impairment was $65 \%$ and $63 \%$, respectively. The cut-off point set for GS was $0,59 \mathrm{~m} / \mathrm{s}$, and for HS was $17.5 \mathrm{~kg}$. This is one of the main contributions of our study based on the fact that we propose a non-cognitive way to classify older adults with cognitive impairment in a national representative sample in a middle-income country, filling knowledge gaps in motor biomarkers of cognitive decline research. Currently, there is no sufficient evidence using GS or HS to identify cognitive impairment, for this reason, many recent publications suggest that combining motor and cognitive measures improves the classification of older adults at risk of dementia $(45,46)$. Around the world, there is an imperious need to find low-cost, accurate, and accessible biomarkers to identify preclinical stages of dementia (47). Therefore, the results of the present study enhance the opportunity of diagnosis in countries without access to expensive biomarkers such as Positron Emision Tomography (PET) imagining. However, further research in this area is needed to extend the generalizability of our findings.

Our study has some limitations. First, the cross-sectional did not make us able to establish causality. This points to the importance of conducting longitudinal studies evaluating the predictive validity of HS and GS and standardizing the optimal cut-off for detecting individuals with impaired cognition. Second, we calculated the ROC curve taking as a comparator a score greater than 24 for the MMSE, which is not validated in our population. GS in the 3 meters test is not a routingly used and this results may underestimated speed(48). However, we do not consider that it biases our results as has been used in previous works(12, 18). Nevertheless, the SABE Colombia study has the largest sample of Latin American older adults, providing to our analyses a good statistical power.

\section{Conclusion}

GS or HS are associated with cognitive impairment, with the larger association in orientation and language domains. GS or HS seems to be useful screening tools for cognitive impairment and can be performed by any clinician. Both motor function tasks share similar operational characteristics and can be used independently. This approach is particularly helpful for low-and middle-income countries such as Colombia since it could reduce the costs associated with a full neuropsychologic assessment or biomarkers such as PET imaging.

\section{Abbreviations}

ROC: receiving operating characteristics, AUC:area under the curve, MCl:mild cognitive impairment, HS:handgrip strength, GS:gait speed, MMSE:mini-mental State Examination, SPPB:short physical performance battery, COPD:chronic obstructive pulmonary disease, SMA:supplementary motor area, PET:positron emision tomography

\section{Declarations}

\section{Ethics approval and consent to participate}


Two universities involved in developing the SABE Colombia study (University of Caldas, ID protocol CBCS-021-14, and University of Valle, ID protocol 09-014 and 011-015) reviewed and approved the study protocol, and written informed consent was obtained from each individual before inclusion and completion of the first examination (including permission to use secondary data and blood samples). The study protocol to the secondary analysis was approved by The Human Subjects Committee at the Pontificia Universidad Javeriana (ACTA ID 20/2017- 2017/180, FM-CIE-0459-17).

\section{Consent for publication}

Not applicable.

\section{Availability of data and materials}

The datasets used and/or analyzed during the current study are available from the corresponding author upon request. Data from SABE 2015 was provided from the Colombian Ministry of Health and Social Protection.

\section{Competing interests}

Authors declare no conflict of interests.

\section{Funding}

This study is part of a larger project that has been funded by Colciencias and the Colombian Ministry of Health and Social Protection -Ministerio de Salud y la Protección Social de Colombia- (The SABE Study ID 2013, no. 764).

Contract of financing RC No. 829 de 2018 Colciencias - Pontificia Universidad Javeriana.

\section{Authors' contributions}

EGC provided the idea and coordinated the project, performed the statistical analysis, interpreted the data and discussed the findings. EGC FBR AJ performed the statistical analysis and analyzed the data. EGC FRV AJ analyzed and interpreted the data. EGC, AJ, IM, GG and MA read and discussed the findings. Al, FL, and CACG were the senior researchers that gave us advice in the composition of the paper

All authors read and approved the final manuscript.

\section{Acknowledgements}

We would like to thank the Aging Institute of the Hospital Universitario San Ignacio for the support that they have given to the Neuroscience and Aging Research Group.

\section{References}

1. International AD. World Alzheimer Report 2019: attitudes to dementia. London Alzheimer's Dis Int. 2019;

2. Lopera F. Clinical Features of Early-Onset Alzheimer Disease in a Large Kindred With an E280A Presenilin-1 Mutation. JAMA J Am Med Assoc. 1997 Mar;277(10):793.

3. Ramirez Aguilar L, Acosta-Uribe J, Giraldo MM, Moreno S, Baena A, Alzate D, et al. Genetic origin of a large family with a novel PSEN1 mutation (lle416Thr). Alzheimer's Dement. 2019;15(5):709-19.

4. Garcia-Cifuentes E, Jaramillo-Jimenez A, Aguillon D, Gómez-Vega M, Velez-Hernandez JE, Cano Gutiérrez C, et al. Prevenir la demencia: un reto para la salud pública en Colombia. Acta Neurológica Colomb. 2019;35(4):208-10.

5. Montero-Odasso MM, Sarquis-Adamson Y, Speechley M, Borrie MJ, Hachinski VC, Wells J, et al. Association of dual-task gait with incident dementia in mild cognitive impairment: Results from the gait and brain study. JAMA Neurol. 2017 Jul;74(7):857-65.

6. Livingston G, Huntley J, Sommerlad A, Ames D, Ballard C, Banerjee S, et al. Dementia prevention, intervention, and care: 2020 report of the Lancet Commission. Lancet. 2020;396(10248):413-46.

7. Buchman AS, Bennett DA. Loss of motor function in preclinical Alzheimer's disease. Vol. 11, Expert Review of Neurotherapeutics. NIH Public Access; 2011. p. 665-76.

8. Beason-Held LL, Goh JO, An Y, Kraut MA, O'Brien RJ, Ferrucci L, et al. Changes in brain function occur years before the onset of cognitive impairment. J Neurosci. 2013;33(46):18008-14.

9. Thal DR, Walter J, Saido TC, Fändrich M. Neuropathology and biochemistry of A 3 and its aggregates in Alzheimer's disease. Vol. 129, Acta Neuropathologica. Springer Verlag; 2015. p. 167-82.

10. Montero-Odasso M, Oteng-Amoako A, Speechley M, Gopaul K, Beauchet O, Annweiler C, et al. The motor signature of mild cognitive impairment: Results from the gait and brain study. Journals Gerontol - Ser A Biol Sci Med Sci. 2014;69(11):1415-21.

11. Garcia-Cifuentes E, David-Pardo DG, Borda MG, Pérez-Zepeda MU, Cano CA. TWO-WAY BRIDGE BETWEEN MUSCULAR DYSFUNCTION AND COGNITIVE IMPAIRMENT: SECONDARY ANALYSIS OF SABE - BOGOTA STUDY. [Internet]. Vol. 6, The Journal of Frailty \& Aging®. Barcelona -Spain; 2017. Available from: http://www.frailty-sarcopenia.com/archives.php 
12. Garcia-Cifuentes E, Márquez I, Vasquez D, Aguillon D, Borda MG, Lopera F, et al. The Role of Gait Speed in Dementia: A Secondary Analysis from the SABE Colombia Study. Dement Geriatr Cogn Disord [Internet]. 2020 Nov 18;1-8. Available from: https://www.karger.com/Article/FullText/510494

13. Ismail Z, Black SE, Camicioli R, Chertkow H, Herrmann N, Laforce R, et al. Recommendations of the 5th Canadian Consensus Conference on the diagnosis and treatment of dementia. Alzheimer's Dement. 2020 Aug;16(8):1182-95.

14. Rijk JM, Roos PRKM, Deckx L, Van den Akker M, Buntinx F. Prognostic value of handgrip strength in people aged 60 years and older: A systematic review and meta-analysis. Geriatr Gerontol Int. 2016 Jan;16(1):5-20.

15. Ishizaki T, Watanabe S, Suzuki T, Shibata H, Haga H. Predictors for functional decline among nondisabled older Japanese living in a community during a 3-year follow-up. J Am Geriatr Soc. 2000 Nov;48(11):1424-9.

16. Fritz NE, McCarthy CJ, Adamo DE. Handgrip strength as a means of monitoring progression of cognitive decline - A scoping review. Vol. 35 , Ageing Research Reviews. Elsevier Ireland Ltd; 2017. p. 112-23.

17. Veronese N, Stubbs B, Trevisan C, Bolzetta F, De Rui M, Solmi M, et al. What physical performance measures predict incident cognitive decline among intact older adults? A 4.4 year follow up study. Exp Gerontol [Internet]. 2016 Aug [cited 2016 Aug 28];81:110-8. Available from: http://www.ncbi.nlm.nih.gov/pubmed/27235850

18. Ramírez-Vélez R, Pérez-Sousa MAM, Venegas-Sanabria LCL, Cano-Gutierrez CA, Hernández-Quiñonez PA, Rincón-Pabón D, et al. Normative Values for the Short Physical Performance Battery (SPPB) and Their Association With Anthropometric Variables in Older Colombian Adults. The SABE Study, 2015. Front Med. 2020;7(February).

19. Mielke M, Roberts R, Savica R, et al. Assessing the temporal relationship between cognition and gait: Slow gait predicts cognitive decline in the Mayo Clinic Study of Aging. J Gerontol A Biol Sci Med Sci. 2013;68A:929-937.

20. Dumurgier J, Artaud F, Touraine C, et al. Gait speed and decline in gait speed as predictors of incident dementia. J Gerontol A Biol Sci Med Sci. 2017;72A:655-661.

21. Buracchio T, Dodge H, Howieson D, Al E. The trajectory of gait speed preceding mild cognitive impairment. Arch Neurol. 2016;67:980-6.

22. Montero-Odasso M. Gait as a biomarker of cognitive impairment and dementia syndromes. Quo vadis? Eur J Neurol. 2016;23(3):437-8.

23. Gomez F, Corchuelo J, Curcio CL, Calzada MT, Mendez F. SABE Colombia: Survey on Health, Well-Being, and Aging in Colombia - Study Design and Protocol [Internet]. Vol. 2016, Current Gerontology and Geriatrics Research. Hindawi; 2016 [cited 2017 Dec 18]. p. 1-7. Available from: https://www.hindawi.com/journals/cggr/2016/7910205/

24. Gomez F, Corchuelo J, Curcio C-L, Calzada M-T, Mendez F. SABE Colombia: Survey on Health, Well-Being, and Aging in Colombia-Study Design and Protocol. Curr Gerontol Geriatr Res [Internet]. 2016 Nov 13 [cited 2017 Dec 18];2016:1-7. Available from:

https://www.hindawi.com/journals/cggr/2016/7910205/

25. Ramírez-Vélez R, Correa-Bautista JE, García-Hermoso A, Cano CA, Izquierdo M. Reference values for handgrip strength and their association with intrinsic capacity domains among older adults. J Cachexia Sarcopenia Muscle. 2019 Apr;10(2):278-86.

26. Mahoney FI BD. FUNCTIONAL EVALUATION: THE BARTHEL INDEX. Md State Med J; 1965. p. 14:61-5.

27. Lawton MP, Brody EM. Assessment of older people: self-maintaining and instrumental activities of daily living. Gerontologist [Internet]. 1969 [cited 2017 Aug 4];9(3):179-86. Available from: http://www.ncbi.nlm.nih.gov/pubmed/5349366

28. Graf C. The Lawton Instrumental Activities of Daily Living (IADL) Scale. AJN. 2008;108(4):59.

29. Folstein MF, Folstein SE, McHugh PR. "Mini-mental”. A practical method for grading the cognitive state of patients for the clinician. J Psychiatr Res. 1975 Nov;12(3):189-98.

30. Creavin ST, Wisniewski S, Noel-Storr AH, Trevelyan CM, Hampton T, Rayment D, et al. Mini-Mental State Examination (MMSE) for the detection of dementia in clinically unevaluated people aged 65 and over in community and primary care populations. Cochrane Database Syst Rev. 2016 Jan; (1):CD011145.

31. Arevalo-Rodriguez I, Smailagic N, Ciapponi A, Sanchez-Perez E, Giannakou A, Roquéi Figuls M, et al. Mini-Mental state examination (MMSE) for the detection of Alzheimer's disease and other dementias in people with mild cognitive impairment (MCl). Cochrane Database Syst Rev. 2013;2013(10).

32. Adamo DE, Anderson T, Koochaki M, Fritz NE. Declines in grip strength may indicate early changes in cognition in healthy middle-aged adults. Wylie GR, editor. PLoS One. 2020 Apr;15(4):e0232021.

33. Moser El, Kropff E, Moser MB. Place cells, grid cells, and the brain's spatial representation system. Annu Rev Neurosci. 2008;31:69-89.

34. Sakurai R, Bartha R, Montero-Odasso M, Newman A. Entorhinal Cortex Volume Is Associated With Dual-Task Gait Cost Among Older Adults With MCl: Results From the Gait and Brain Study. J Gerontol A Biol Sci Med Sci [Internet]. 2018;00(00):1-7. Available from:

https://watermark.silverchair.com/gly084.pdf?

token=AQECAHi208BE490oan9kkhW_Ercy7Dm3ZL_9Cf3qfKAc485ysgAAAdMwggHPBgkqhkiG9w0BBwagggHAMIIBvAIBADCCAbUGCSqGSIb3DQEHATA $\epsilon$

35. Beauchet O, Allali G, Annweiler C, Bridenbaugh S, Assal F, Kressig RW, et al. Gait Variability among Healthy Adults: Low and High Stride-to-Stride Variability Are Both a Reflection of Gait Stability. Gerontology. 2009 Nov;55(6):702-6.

36. Beauchet O, Annweiler C, Montero-Odasso M, Fantino B, Herrmann FR, Allali G. Gait control: A specific subdomain of executive function? J Neuroeng Rehabil. 2012;9(1):1-5.

37. Hausdorff JM, Yogev G, Springer S, Simon ES, Giladi N. Walking is more like catching than tapping: Gait in the elderly as a complex cognitive task. Exp Brain Res. 2005 Jul;164(4):541-8.

38. MacAulay RK, Wagner MT, Szeles D, Milano NJ. Improving Sensitivity to Detect Mild Cognitive Impairment: Cognitive Load Dual-Task Gait Speed Assessment. J Int Neuropsychol Soc. 2017;23(6):493-501. 
39. Reilly J, Rodriguez AD, Lamy M, Neils-Strunjas J. Cognition, language, and clinical pathological features of non-Alzheimer's dementias: An overview. J Commun Disord. 2010;43(5):438-52.

40. Hertrich I, Dietrich S, Ackermann H. The role of the supplementary motor area for speech and language processing. Vol. 68 , Neuroscience and Biobehavioral Reviews. Elsevier Ltd; 2016. p. 602-10.

41. Vaz-Patto M, Bueno B, Ribeiro Ó, Teixeira L, Afonso RM. Association between handgrip strength, walking, age-related illnesses and cognitive status in a sample of Portuguese centenarians. Eur Rev Aging Phys Act. 2017 Jul;14(1).

42. Chou MY, Nishita Y, Nakagawa T, Tange C, Tomida M, Shimokata H, et al. Role of gait speed and grip strength in predicting 10-year cognitive decline among community-dwelling older people. BMC Geriatr. 2019 Jul;19(1):186.

43. Inzitari M, Newman AB, Yaffe K, Boudreau R, De Rekeneire N, Shorr R, et al. Gait speed predicts decline in attention and psychomotor speed in older adults: The health aging and body composition study. Neuroepidemiology. 2008;29(3-4):156-62.

44. Jin Y, Sumsuzzman DM, Choi J, Kang H, Lee SR, Hong Y. Molecular and functional interaction of the myokine irisin with physical exercise and Alzheimer's disease. Molecules. 2018;23(12):1-13.

45. Ayers E, Verghese J. Gait Dysfunction in Motoric Cognitive Risk Syndrome. J Alzheimer's Dis. 2019;71(s1):S95-103.

46. Montero-Odasso M, Speechley M, Muir-Hunter SW, Pieruccini-Faria F, Sarquis-Adamson Y, Hachinski V, et al. Dual decline in gait speed and cognition is associated with future dementia: evidence for a phenotype. Age Ageing [Internet]. 2020 Jun 18;(June). Available from:

https://academic.oup.com/ageing/advance-article/doi/10.1093/ageing/afaa106/5857260

47. Aisen PS, Cummings J, Jack CR, Morris JC, Sperling R, Frölich L, et al. On the path to 2025: Understanding the Alzheimer's disease continuum. Alzheimer's Res Ther. 2017;9(1):1-10.

48. Krumpoch S, Lindemann U, Rappl A, Becker C, Sieber CC, Freiberger E. The effect of different test protocols and walking distances on gait speed in older persons. Aging Clin Exp Res [Internet]. 2020;(0123456789). Available from: https://doi.org/10.1007/s40520-020-01703-z

\section{Tables}

Due to technical limitations, table 3 is only available as a download in the Supplemental Files section.

\section{Figures}

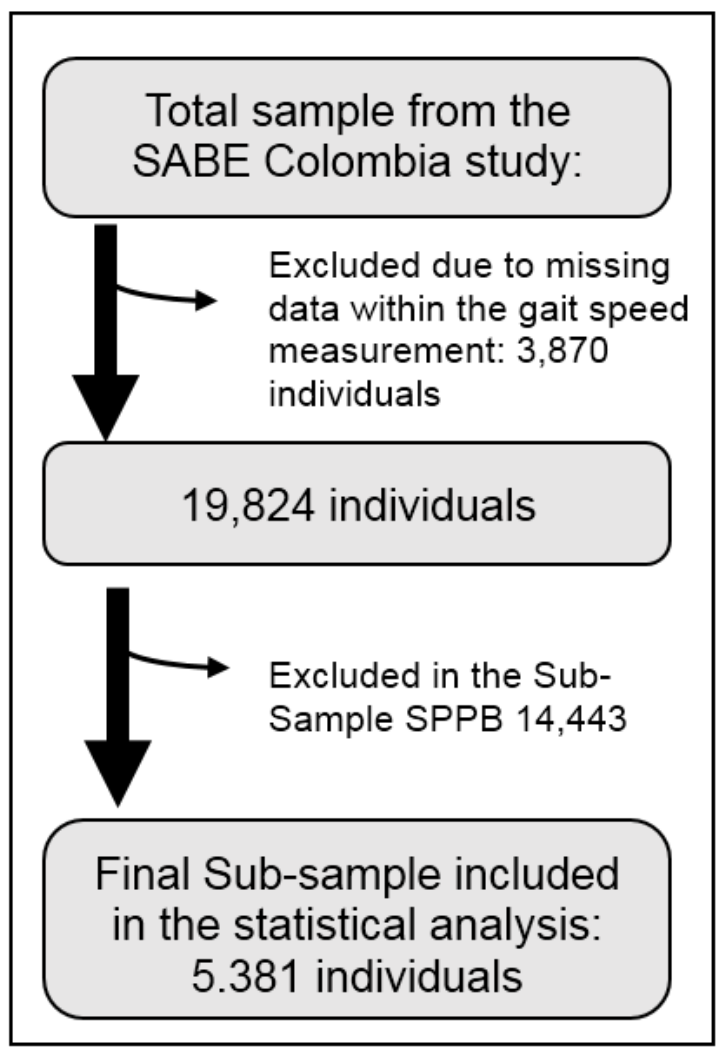

Figure 1

Flow-chart selection. 

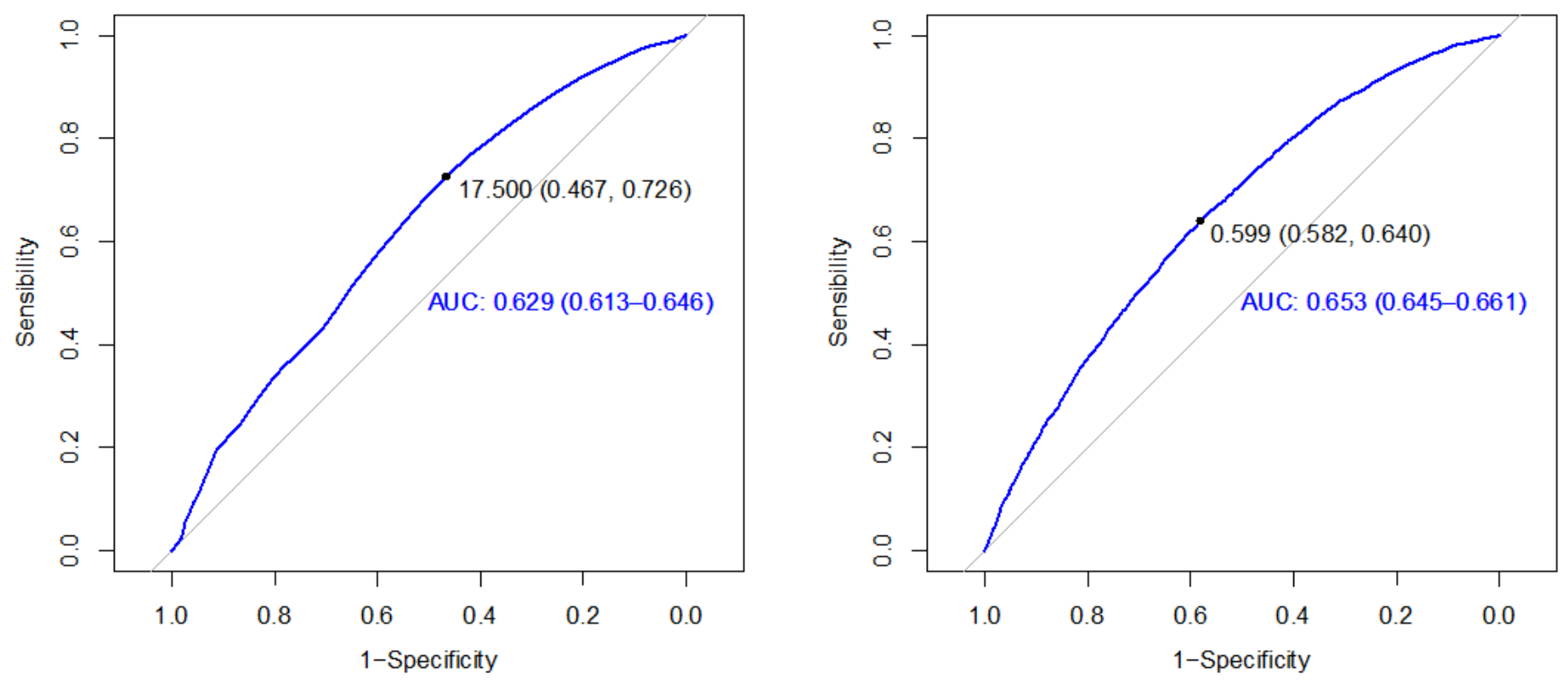

Figure 2

ROC curves defining cut-off points for HS (left) and GS (right) as markers for cognitive impairment, in the points with largest AUC. AUC: area under the curve, HS: handgrip strength, GS: gait speed

\section{Supplementary Files}

This is a list of supplementary files associated with this preprint. Click to download.

- TablesMuscularFunction.xlsx 